\title{
EFFECTS OF METALLICITY AND AGN ACTIVITY ON THE MID-INFRARED DUST EMISSION OF GALAXIES
}

\author{
Hong $\mathrm{Wu}^{1,2}$, Yi-Nan Zhu ${ }^{1,3}$, Chen $\mathrm{Cao}^{1,3}$, Bo Qin ${ }^{1}$ \\ hwu@bao.ac.cn
}

\begin{abstract}
Using a sample of the Spitzer SWIRE-field galaxies whose optical spectra are taken from the Data Release 4 of the Sloan Digital Sky Survey, we study possible correlations between the Mid-InfraRed (MIR) dust emission from these galaxies and both their metallicities and AGN activities. We find that both metallicity and AGN activity are well correlated with the following ratios: PAH(Polycyclic Aromatic Hydrocarbon)-to-star, VSG(Very Small Grain)-to-star and PAH-to-VSG, which can be characterized by $\nu L_{\nu}\left[8 \mu m\right.$ (dust)] $/ \nu L_{\nu}[3.6 \mu m]$, $\nu L_{\nu}[24 \mu m] / \nu L_{\nu}[3.6 \mu m]$, and $\nu L_{\nu}[8 \mu m$ (dust) $] / \nu L_{\nu}[24 \mu m]$ respectively. We argue that our MIR-metallicity correlation could be explained by either the amount of dust (ongoing dust formation) or dust destruction (PAHs and VSGs could be destroyed by hard and intense radiation fields), and that the MIR-AGN correlation could arise due to either PAH destruction or an enhanced VSG continuum by the central AGN.
\end{abstract}

Subject headings: infrared: galaxies - galaxies: starburst - galaxies: abundances - galaxies: active

\section{INTRODUCTION}

In galaxies, the MIR bands not only contain a large number of atomic, ionic and molecular lines, but also cover various dust features from grains of different size (Sturm et al. 2002).

\footnotetext{
${ }^{1}$ National Astronomical Observatories, Chinese Academy of Sciences, A20 Datun Road, Beijing 100012, P.R. China

${ }^{2}$ Visiting Scholar, Institute for Astronomy, University of Hawaii, 2680 Woodlawn Drive, Honolulu, HI 96822

${ }^{3}$ Graduate School, Chinese Academy of Sciences, Beijing, 100039, P.R. China
} 
These include the features related to the polycyclic aromatic hydrocarbons (PAHs) and the dust continuum from the very small grains (VSGs). Recent work (Calzetti et al. 2005; Wu et al. 2005; Vogler et al. 2005; Alonso-Herrero et al. 2006; Elbaz et al. 2002; Boselli et al. 2004; Förster Schreiber et al. 2004; Roussel et al. 2001) have shown that the MIR dust emission are well correlated with the star formation rate (SFR) indicators, such as $\mathrm{H} \alpha, \mathrm{Pa} \alpha$, radio, UV, and far-infrared in either star forming regions in galaxies or galaxies themselves as a whole. With further advantages of having less extinction, the MIR dust luminosities are ideal tracers of star formation and can be widely used to estimate the SFR.

The MIR emission in galaxies could, however, be affected by many factors. For example, early observations on the ground as well as recent space observations by the Infrared Space Observatory (ISO) and the Spitzer Space Telescope (Werner et al. 2004) have shown that the PAH emission is generally suppressed in low-metallicity galaxies (Roche et al. 1991; Madden 2000; Galliano et al. 2005; Madden et al. 2006; Wu et al. 2006), or even missing in the most metal-poor galaxies (Houck et al. 2004; Engelbracht et al. 2005; Thuan et al. 1999). Siebenmorgen et al. (2004) have demonstrated the absence of PAHs in the nuclei of AGN-hosting galaxies. Using the Spitzer Infrared Spectrograph (IRS, Houck et al. 2004) spectra, Weedman et al. (2005) have confirmed that the PAH emission is much weaker in AGNs. Moreover, the AGN activity could also steepen the shape of the MIR VSG continuum in some active galaxies (Verma et al. 2005).

To quantitatively examine the factors that could affect the MIR emission in galaxies, we need a sample of galaxies with both MIR measurements and optical spectra. The Spitzer Wide-area InfraRed Extragalactic survey (SWIRE; Lonsdale et al. 2003) and the Sloan Digital Sky Survey (SDSS; Stoughton et al. 2002) provide such opportunities. The Infrared Array Camera (IRAC, Fazio et al. 2004) $8 \mu \mathrm{m}$ band, which just covers the main PAH features, can be used to trace the PAH emission, while the Multiband Imaging Photometer (MIPS, Rieke et al. 2004) $24 \mu \mathrm{m}$ band, which covers the continuum, can trace the VSG emission. The SDSS spectra provide us information of both the metallicities and AGN activities of galaxies. In the present paper, we mainly focus on the correlations between the MIR emission and metallicity/AGN-activity.

In Section 2, we describe the data, data reduction, and the sample. The spectral classification, the metallicity and the definition of AGN activity are presented in Section 3. The correlation analysis between the MIR and metallicity/AGN-activity are shown in Section 4. Finaly, Section 5 and 6 are discussions and summary. Throughout the paper we adopt a $\Lambda \mathrm{CDM}$ cosmology with $\Omega_{M}=0.3, \Omega_{\Lambda}=0.7$, and $H_{0}=70$. 


\section{DATA AND SAMPLE}

The Spitzer SWIRE fields (Lonsdale et al. 2003) of ELAIS-N1, ELAIS-N2, and Lockman Hole have been imaged by both instruments: IRAC (3.6, 4.5, 5.8, $8.0 \mu \mathrm{m}$; Fazio et al. 2004) and MIPS (24 $\mu \mathrm{m}$; Rieke et al. 2004). These fields have also been completely or partly covered by the SDSS Data Release 4 (DR4; Adelman-McCarthy et al. 2006). The overlap area between the three SWIRE fields and the SDSS DR4 field used in this paper is about 15 degree $^{2}$. The Basic Calibrated Data (BCD) images of the IRAC four bands were obtained from the Spitzer Science Center, which include flat-field corrections, dark subtraction, linearity and flux calibrations (Fazio et al. 2004). The IRAC images (in all four IRAC bands) were mosaiced from the BCD images after pointing refinement, distortion correction and cosmic-ray removal with a final pixel scale of $0.6^{\prime \prime}$ as described by Huang et al. (2004) and Wu et al. (2005), whilst the MIPS $24 \mu \mathrm{m}$ images were in a similar way but with a final pixel scale of 1.225" (Wen et al. 2007; Cao \& Wu 2007). The FWHMs of point spread functions (PSFs) in mosaiced images are around $2^{\prime \prime}$ for the IRAC four bands and 6 " for the MIPS $24 \mu \mathrm{m}$ band. Matching the sources detected by the SExtractor (Bertin \& Arnouts 1996) in all the five bands with the 2MASS sources (Cutri et al. 2003) ensured the astrometric uncertainties to be less than $0.1^{\prime \prime}$.

The MIR photometries were obtained by the SExtractor with an aperture of $6^{\prime \prime}$ for the IRAC four bands and the MIPS $24 \mu \mathrm{m}$ band. The apertures of $6^{\prime \prime}$ were used to match the FWHM of the PSF of the MIPS $24 \mu \mathrm{m}$ band, though the fiber aperture of the SDSS spectra is $3^{\prime \prime}$. All these magnitudes are in the AB magnitude system (Oke \& Gunn 1983). The values of $-0.20,-0.17,-0.23,-0.41$ magnitudes for $3.6,4.5,5.8,8.0 \mu \mathrm{m}$ bands were adopted to correct the apertures of $6^{\prime \prime}$ to $24^{\prime \prime}$ respectively. Comparing with the model colors of the IRAC magnitudes and 2MASS $K_{S}$ magnitude (Cutri et al. 2003) for the stars with $J-K_{S}$ $\leq 0.3$ as Lacy et al. (2005) did for sources in the Spitzer extragalactic First Look Survey (xFLS) field, small additional corrections were made for all the four IRAC bands. And these give us the calibration errors in the four IRAC bands better than 0.08 mag. A factor of -1.21 magnitude was adopted to correct the aperture from $6^{\prime \prime}$ to $30^{\prime \prime}$ for the $24 \mu \mathrm{m}$ band. An additional correction of $-0.196 \mathrm{mag}$ was also performed for the $24 \mu \mathrm{m}$ sources. This included a factor of 1.15 of calibration and a factor of 1.04 of color correction (Surace et al. 2005). The calibration accuracy of the $24 \mu \mathrm{m}$ photometry is better than $10 \%$ (Rieke et al. 2004).

To build our galaxy sample, we first selected the galaxies of three SWIRE fields from the main galaxy sample of the SDSS-DR4 (Adelman-McCarthy et al. 2006) and then matched them with the MIR sources detected in the Spitzer SWIRE fields with a matching radius of $2^{\prime \prime} .14$ out of 1628 (about 1\%) of the SDSS galaxies were mis-matched with the MIR sources, due to the $2^{\prime \prime}$ matching radius. To obtain reliable line ratios and to avoid possible 
contaminations from the old stellar population, we only kept the sources with a reliable $\mathrm{H} \beta$ flux $(\mathrm{S} / \mathrm{N} \geq 5)$ and a higher $\mathrm{H} \alpha$ equivalent width (less than -5.0 , the minus here means emission), where the $\mathrm{H} \alpha$ line emission flux used here was from BC03 (Bruzual \& Charlot 2003) continuum-subtracted. 1 This gives our sample of 600 galaxies. All these galaxies have $8 \mu \mathrm{m}$ fluxes, and most have $24 \mu \mathrm{m}$ fluxes. The redshifts of the galaxies in the sample are from 0.004 to 0.18 .

\section{SPECTRAL CLASSIFICATION, METALLICITY AND AGN ACTIVITY}

Our spectral classification was based on the traditional line-diagnostic diagram [OIII]/H $\beta$ versus [NII]/H $\alpha$, Veilleux \& Osterbrock 1987) as in Figure1, The dotted curve in the figure is the parametrized curve by Kauffmann et al. (2003) while the dashed curve is the theoretical boundary for starburst by Kewley et al. (2001). The galaxies below the dotted curve are HII galaxies, the galaxies between the dotted curve and dashed curve are mixture types (Wu et al. 1998) or composites (Kewley et al. 2006), and galaxies above the dashed curve are narrow line AGNs. There are altogether 472 HII galaxies, 99 composites and 29 AGNs.

The metallicities of HII galaxies were from the metallicity catalog of SDSS DR4 (Tremonti et al. 2004) 2. Here we adopted the median of the likelihood distribution for $12+\mathrm{Log} \mathrm{O} / \mathrm{H}$. 329 HII galaxies, which have measured metallicities in the Tremonti et al. (2004) catalog, were selected for the following analysis. 297 of them have $24 \mu \mathrm{m}$ fluxes. We defined dwarf galaxies as the galaxies whose absolute B magnitudes were greater than -18 . Here the B magnitudes were calculated from the SDSS $g$ - and $r$ - magnitudes (Smith et al. 2002). The dwarf galaxies are marked with pluses in Figure 1.

Figure 2 shows the metallicities of HII galaxies as a function of the internal reddening. The internal reddening (characterized by $\mathrm{E}(\mathrm{B}-\mathrm{V})$ ) was derived from the Balmer decrement $\mathrm{H} \alpha / \mathrm{H} \beta$ (Calzetti 2001). The figure suggests that dusty galaxies tend to be metal rich.

To quantify AGN activity, here we define a distance $d_{A G N}$ in dex (see the red dotted line in Figure 1 as an example) in the traditional line-diagnostic diagram $[\mathrm{OIII}] / \mathrm{H} \beta$ versus $[\mathrm{NII}] / \mathrm{H} \alpha$ to represent AGN activity. This is quite similar to the star-forming distance defined by Kewley et al. (2006) in the diagnostic diagram $[\mathrm{OIII}] / \mathrm{H} \beta$ versus $[\mathrm{OI}] / \mathrm{H} \alpha$ or $[\mathrm{SII}] / \mathrm{H} \alpha$. Considering that the composites could be dominated by star formation and that this would

\footnotetext{
${ }^{1} \mathrm{MPA}$ emission line catalog, which is available at http://www.mpa-garching.mpg.de/SDSS/DR4/raw data.html

${ }^{2}$ Available at http://www.mpa-garching.mpg.de/SDSS/DR4/Data/oh catalogue.html
} 
weaken possible contributions from the central AGN, the $d_{A G N}$ is defined as the distance of an AGN from its position $\left(x_{p}, y_{p}\right)$ to the Kewley et al. (2001)'s curve along the parallel of the best linear fitting of all AGNs (solid line) in Figure 1, and can be formulized as :

$$
\begin{aligned}
& d_{A G N}=0.507\left(5.8 x_{p}+y_{p}-3.196+A\right) \\
& A=\sqrt{14.152+\left(y_{p}-5.8 x_{p}+1.536\right)^{2}},
\end{aligned}
$$

where $x=\log [\mathrm{NII}] / \mathrm{H} \alpha$ and $y=\log [\mathrm{OIII}] / \mathrm{H} \beta$. Larger distances correspond to stronger AGN activities. After removing the two most deviated AGNs, 27 AGNs between the two dash-dotted lines were selected for our following analysis. Three of them do not have $24 \mu \mathrm{m}$ fluxes.

\section{ANALYSIS}

\subsection{MIR Ratios Versus Metallicity}

To correct the redshift effect, we adopted the SED of the normal HII galaxy NGC 3351 from IRS observations in the Spitzer Legacy Program SINGS (Kennicutt et al. 2003) as the template for the K-corrections for our sample galaxies in both the $24 \mu \mathrm{m}$ and $8 \mu \mathrm{m}$ bands (Wu et al. 2005). In order to avoid the strong PAH emission, we used the $3.6 \mu \mathrm{m}$ band to estimate the stellar contribution in the $8.0 \mu \mathrm{m}$ band. A factor of 0.26 (Wu et al. 2005) was used to scale the stellar continuum of $3.6 \mu \mathrm{m}$ to that of $8 \mu \mathrm{m}$. After subtracting the corresponding stellar continuum contribution, we obtained the flux of $8 \mu \mathrm{m}$ dust emission denoted by $8 \mu \mathrm{m}$ (dust), as in Wu et al. (2005). The $8 \mu \mathrm{m}$ (dust) includes the dust emission from both the PAHs and VSGs. Considering that the contribution of the stellar continuum in the $24 \mu \mathrm{m}$ band is quite small and negligible, we have not removed the stellar continuum from the $24 \mu \mathrm{m}$ band flux.

It is well known that the mass of a galaxy is well related to its metallicity (Pagel \& Edmunds 1981). To avoid possible effects caused by different mass variations, we used the MIR ratios $\nu L_{\nu}[8 \mu m(d u s t)] / \nu L_{\nu}[3.6 \mu m]$ and $\nu L_{\nu}[24 \mu m] / \nu L_{\nu}[3.6 \mu m]$ instead of the MIR luminosities in our analysis. These ratios can be explained as the MIR dust emission in mass unit (Wen et al. 2007), since the $3.6 \mu \mathrm{m}$ luminosity can approximately represent the stellar mass (Smith et al. 2007a; Hancock et al. 2007).

Figure 3 shows the MIR ratios $\nu L_{\nu}[8 \mu m(d u s t)] / \nu L_{\nu}[3.6 \mu m], \nu L_{\nu}[24 \mu m] / \nu L_{\nu}[3.6 \mu m]$, and $\nu L_{\nu}[8 \mu m(d u s t)] / \nu L_{\nu}[24 \mu m]$ as functions of metallicity in the log-log space. We plotted the mean values (in diamonds) and the $1-\sigma$ standard deviation bars with a metallicity bin 
of 0.2. The plus symbols represent the dwarf galaxies. We can see that the MIR ratios and metallicity are correlated. Both $\nu L_{\nu}[8 \mu m(d u s t)] / \nu L_{\nu}[3.6 \mu m]$ and $\nu L_{\nu}[24 \mu m] / \nu L_{\nu}[3.6 \mu m]$ ratios show nearly linear correlations with metallicity in the log-log space, and the Spearman correlations are tight with the probabilities of null-hypothesis being $2.7 \times 10^{-17}$ and $5.2 \times 10^{-7}$ respectively. Almost all the dwarf galaxies here obey the same correlation as the normal galaxies do, except Mrk1434 which has the lowest metallicity in our sample. Although the MIR ratio $\nu L_{\nu}[8 \mu m(d u s t)] / \nu L_{\nu}[24 \mu m]$ also correlates with metallicity, this correlation only exists for galaxies with metallicities lower than 8.7. For galaxies with higher metallicities, the $\nu L_{\nu}[8 \mu m($ dust $)] / \nu L_{\nu}[24 \mu m]$ ratios almost remain constant. Compared with the above two correlations, the Spearman correlation between $\nu L_{\nu}[8 \mu m(d u s t)] / \nu L_{\nu}[24 \mu m]$ and metallicity is much weaker, with the probability of null-hypothesis being 0.02 .

\subsection{MIR Ratios Versus AGN Distance $d_{A G N}$}

Figure 4 shows the MIR ratios as functions of AGN activity. Here we used the distance $d_{A G N}$ as defined in Section 3 to characterize AGN activity. The MIR ratio $\nu L_{\nu}[8 \mu m(d u s t)] / \nu L_{\nu}[3.6 \mu m]$ seems to mildly increase with the distance $d_{A G N}$. The probability of null-hypothesis of the Spearman correlation is 0.3 , indicating that the correlation is poor. The ratio $\nu L_{\nu}[24 \mu m] / \nu L_{\nu}[3.6 \mu m]$ is well correlated with $d_{A G N}$. The probability of null-hypothesis of such a correlation is $3.6 \times 10^{-4}$, indicating that the $24 \mu \mathrm{m}$ emission in mass unit tend to be stronger with the level of AGN activity. On the contrary, the MIR ratio $\nu L_{\nu}[8 \mu m(d u s t)] / \nu L_{\nu}[24 \mu m]$ is anticorrelated with $d_{A G N}$. The probability of null-hypothesis is $1.7 \times 10^{-5}$. The correlation between $\nu L_{\nu}[8 \mu m(d u s t)] / \nu L_{\nu}[24 \mu m]$ and $d_{A G N}$ is the tightest among the three. Even the most discrepant point in Figure 4 (a) and (b) also follows such a correlation.

\subsection{MIR Ratios Versus Internal Reddening}

To explore possible influence of the internal reddening on the MIR emission, the MIR ratios were also plotted against E(B-V) in Figure 5. For HII galaxies, both ratios, $\nu L_{\nu}[8 \mu m(d u s t)] / \nu L_{\nu}[3.6 \mu m$ and $\nu L_{\nu}[24 \mu \mathrm{m}] / \nu L_{\nu}[3.6 \mu \mathrm{m}]$, show tight correlations with $\mathrm{E}(\mathrm{B}-\mathrm{V})$. The probabilities of nullhypothesis of the Spearman correlation are $3.1 \times 10^{-22}$ and $3.4 \times 10^{-10}$ respectively. Both correlations indicate that the dusty galaxies have relatively strong $8 \mu \mathrm{m}$ or $24 \mu \mathrm{m}$ dust emission. Such correlations can also be expected in Figure 3, since the metallicities of HII galaxies are also correlated with their internal reddening (Figure[2). However, $\nu L_{\nu}[8 \mu m(d u s t)] / \nu L_{\nu}[24 \mu m]$ does not show any correlation with $\mathrm{E}(\mathrm{B}-\mathrm{V})$. The probability of null-hypothesis is 0.16 . Therefore, this ratio does not depend on the internal reddening. 
For AGNs, all the three MIR ratios do not correlate with reddening. The Spearman correlations show that the probabilities of null-hypothesis are 1.00, 0.16, and 0.09 respectively, indicating that the internal reddening does not affect all the three MIR ratios of AGNs.

\section{DISCUSSIONS}

\subsection{Aperture Effect}

Since the galaxies in our sample cover a redshift range from 0.004 to 0.17 , the 6 " aperture corresponds to a physical size from several hundred pc to several tens kpc. Will the aperture affect the correlations we obtained? We examined this by grouping the galaxies into several redshift bins, as shown in Figure 3 and Figure 4 . We find that either the HII galaxies (Figure 3) or AGNs (Figure 4) with different redshifts follow the same correlations.

To further explore the aperture affect, we plotted the MIR ratios as functions of redshift in Figure6. The mean MIR ratios (except for dwarf galaxies) and the corresponding standard deviations with a redshift bin of 0.02 were also plotted. There is only a small variation of the mean MIR ratios (less than 0.2 dex) with redshift.

Meanwhile, we "virtually" placed a template galaxy (e.g., the SINGS galaxy NGC 3351) in different redshifts. The $6^{\prime \prime}$ aperture corresponds to different physical sizes at different redshifts. Therefore, we did the aperture photometry for the MIR images of the SINGS galaxy NGC 3351 with a set of apertures, whose physical sizes are equal to those of the $6^{\prime \prime}$ aperture at different redshifts. As a result, we can obtain the MIR ratios of NGC 3351 as functions of redshift or aperture, which are plotted as the dotted curves in Figure 6. Here we only gave the MIR ratios of NGC 3351 at the rest-frame and do not consider the Kcorrection. From the figure, all the three MIR ratios of the template NGC 3351 only vary by about 0.1 to 0.2 dex with redshift.

Both the sample statistics and template modelling show that the aperture effect on the MIR ratios is weaker than the correlations shown in Figure 3, Therefore, we believe that the aperture effect would not significantly affect our correlations obtained from Figure 3 and Figure 4 .

\subsection{Metallicity and Dust Emission}

Although the VSG continuum also contributes to the Spitzer $8 \mu \mathrm{m}$ band (Smith et al. $2007 \mathrm{~b})$, in HII galaxies, the strongest $7.7 \mu \mathrm{m}$ PAH features still dominate the emission in 
this band. However, the VSG continuum dominates the Spitzer MIPS $24 \mu \mathrm{m}$ band. Therefore, the correlations between the MIR ratios and metallicity in Figure 3 reveal the relationships between the ratios of PAH-to-star/VSG-to-star/PAH-to-VSG and metallicity. Here, the $3.6 \mu \mathrm{m}$ luminosity can approximately represent the stellar mass (Smith et al. 2007a; Hancock et al. 2007). All these ratios increase with metallicity, though the details are different (e.g., different slopes). Apparently the dust properties in the HII galaxies seem to depend on metallicity. However, the metallicity itself is often related to many other factors, such as mass (the mass-metallicity relation, i.e., lower mass dwarf galaxies often have lower metallicities), and radiation field (in a low metallicity environment, the radiation field is often hard). Hence, it is necessary to explore the underlying mechanisms.

In fact, all these MIR behaviors mainly depend on two main factors: the local radiation field and the dust. The properties of the radiation field include the hardness, which can be characterized by the MIR [NeIII]/[NeII] (Thornley et al. 2000; Wu et al. 2006; Madden et al. 2006), and the intensity, which can be characterized by either the MIR luminosity density (Engelbracht et al. 2005; Wu et al. 2006) or star formation rate (Rosenberg et al. 2006). The very hard and intense radiation field could destroy the PAHs (Galliano et al. 2005), and even the VSGs (Contursi et al. 2000).

The dust properties include the amount of dust, and the fractions and spatial distributions of the PAHs and VSGs. Galaxies with lower or vanishing PAH or VSG emission could contain less amount of dust, possibly because they have low metallicities, which lack material to form dust, or their masses are too low to retain the dust against the radiation pressure and winds, or they are too young, to have had enough time to form PAHs (Hogg et al. 2005; Madden et al. 2006; Draine et al. 2007). Different fractions and distributions of the PAHs and VSGs could result in different PAH-to-VSG ratios.

The positive correlations between the PAH/VSG-to-star ratios and metallicity obtained from Figure 3 could be explained by the amount of dust. It could be expected from the relations between the reddening and metallicity (Figure 21) or the PAH/VSG-to-star ratios (Figure 5 (a) and (b)). In fact, the internal reddening is directly related to the amount of dust if assuming a constant dust-to-gas ratio, due to the tight correlation between $\mathrm{E}(\mathrm{B}-\mathrm{V})$ and H column density (Bohlin et al. 1978; Draine 2003). Therefore, the low PAH/VSG-to-star ratio of lower metallicity galaxies could not be due to that their masses are too low to retain the amount of dust, because some of the normal galaxies also follow the same correlation as lower metallicity galaxies do in Figure 3. It could be that they are so young that a large amount of dust has not had enough time to be released. This is supported by Figure 2 .

As for the PAH-to-VSG ratio, if assuming that the AGB stars are the dominant carbon reservoir to form the PAHs (Dwek 1998) with an age of star formation less than 500Myr 
when the $4 \mathrm{M} \odot$ has entered the AGB stage(Madden et al. 2006), the PAH-to-VSG ratio could increase with time. After that, the ratio could approach a constant. This seems to be consistent with Figure 3(c), in which the value of $\nu L_{\nu}[8 \mu m(d u s t)] / \nu L_{\nu}[24 \mu m]$ increases with metallicity and then remains constant beyond solar metallicity. Such an explanation is quite similar to the explanation of the low dust-to-gas ratios for low metallicity SINGS galaxies by Draine et al. (2007). Also the behavior of the PAH-to-VSG ratio with time can be well described by the Figure 4 of Galliano (2006) recent work on chemical evolution modeling of carbon and silicate grains.

Another explanation could be that the hard and intense radiation field from the young stars in the lower metallicity galaxies can destroy not only the PAHs but also the VSGs. Since the PAHs are much more sensitive to hard radiation field than the VSGs are, the slope of the PAH-metallicity relation is much steeper than that of the VSGs. This results in a decreasing PAH-to-VSG ratio in the lower metallicity galaxies (Figure [3). Such a destruction effect is further supported by Figure 7 , which shows the anti-correlation between $\nu L_{\nu}[8 \mu m($ dust $)] / \nu L_{\nu}[24 \mu m]$ and the $24 \mu \mathrm{m}$ intensity for the sample of HII galaxies, though such an anti-correlation is not as steep as that for AGNs. The $24 \mu \mathrm{m}$ intensity is obtained from the ratio of the $24 \mu \mathrm{m}$ luminosity to the physical area in the aperture of $66^{\prime \prime}$.

We thus conclude that both effects of dust formation and destruction can explain the correlations between the MIR ratios and metallicity. This is consistent with the conclusions for PAHs by Wu et al. (2006) and Draine \& Li (2007).

\subsection{AGN Activity and Dust Emission}

The MIR spectra of AGNs were already noted to be void of PAH features (Roche \& Aitken 1985; Roche et al. 1991; Aitken \& Roche 1985; Genzel \& Cesarsky 2000; Laurent et al. 2000; Siebenmorgen et al. 2004). This could be explained by the destruction of PAHs due to the hard radiation field of the central source (Madden et al.|2006). It is also supported by recent Spitzer results given by Dale et al. (2006) and Armus et al. (2007), who used [OIV]/[NeII] as the indicator of the hardness of radiation field. However, Figure 4(a) shows that the PAHto-star ratio apparently does not have any tendency to decrease but rather increases with AGN activity. Draine et al. (2007) explained the SINGS AGNs as low-luminosity AGNs, which have little effect on the $8 \mu \mathrm{m}$ dust emission so that they show no evidence of PAH suppression. This is however not the case for our sample of AGNs, since all of them have higher $\mathrm{H} \alpha$ equivalent widths according to our selection in Section 2. The amount of dust cannot account for that, since the internal reddening does not correlate with any of the three MIR ratios (Figure 5). We suggest that the AGNs not only can destroy the PAHs in the central 
regions but could also possibly excite the PAH emission in the outer regions (Smith et al. $2007 \mathrm{~b})$.

Another explanation is that the central AGN raises the level of the VSG continuum in the $8 \mu \mathrm{m}$ band. Similarly the sharp increase of the VSG-to-star ratio with AGN activity in Figure 4(b) may also be due to the enhanced $24 \mu \mathrm{m}$ VSG continuum by the powerful AGN. Although the outer PAH emission could be excited by the central AGN, both the destruction of PAHs and the enhancement of the $24 \mu \mathrm{m}$ VSG continuum by the AGN would result in the tight anti-correlation between the PAH-to-VSG ratio and AGN activity.

\subsection{Radiation Field and Dust Emission}

One of the important factors in explaining the correlations between the MIR ratios and metallicity or AGN activity is the radiation field, which could be provided by either young stars or AGNs. Hogg et al. (2005) suggested that [OIII]/H $\beta$ could be an indicator of radiation field hardness.

Figure 8 shows the three MIR ratios as functions of $[\mathrm{OIII}] / \mathrm{H} \beta$. For AGNs, the correlations between the MIR ratios and $[\mathrm{OIII}] / \mathrm{H} \beta$ are similar to those between the MIR ratios and $d_{A G N}$. So, the AGN distance $d_{A G N}$ is directly related to the radiation field. For HII galaxies, all these MIR ratios show the anti-correlation with $[\mathrm{OIII}] / \mathrm{H} \beta$. With $[\mathrm{OIII}] / \mathrm{H} \beta$ increasing, the PAH/VSG-to-star ratios of the HII galaxies and AGNs show different features. Hence, the PAH/VSG-to-star ratios seem to depend on not only the radiation field, but also some other important factor as well. As discussed above, in the HII galaxies. the amount of dust would play an important role. However the PAH-to-VSG ratio, which describes the fraction of two different dust components, does not depend on the amount of dust but rather the radiation field. So, the PAH-to-VSG ratio of the HII galaxies and AGNs would present the similar behaviors along $[\mathrm{OIII}] / \mathrm{H} \beta$, as shown in Figure 8 (c).

In a hard radiation field environment $([\mathrm{OIII}] / \mathrm{H} \beta>1)$, the PAH-to-VSG ratio decreases sharply with increasing $[\mathrm{OIII}] / \mathrm{H} \beta$, whether in HII galaxies or in AGNs. However, the PAHto-VSG ratio remains constant in a weak radiation field environment $([\mathrm{OIII}] / \mathrm{H} \beta<1)$. We conclude that the hard radiation field, rather than metallicity or AGN activity, is the most direct factor to determine the PAH-to-VSG ratio. 


\section{SUMMARY}

We have constructed a sample of galaxies from both the SDSS Data Released 4 and the Spitzer SWIRE fields to study possible effects of both metallicity and AGN activity on the MIR dust emission. Based on this sample we have found that:

The MIR ratios: PAH-to-star, VSG-to-star and PAH-to-VSG, which can be characterized respectively by $\nu L_{\nu}[8 \mu m(d u s t)] / \nu L_{\nu}[3.6 \mu m], \nu L_{\nu}[24 \mu m] / \nu L_{\nu}[3.6 \mu m]$, and $\nu L_{\nu}[8 \mu m(d u s t)] / \nu L_{\nu}[24 \mu m]$, are found to be positively correlated with the metallicities of galaxies. The above correlations could be explained by either the amount of dust (ongoing dust formation) or dust destruction (either the PAHs or VSGs could be destroyed by the hard and strong radiation field).

The VSG-to-star and PAH-to-VSG ratios are strongly correlated or anti-correlated with AGN activity. However, the PAH-to-star ratio poorly depends on AGN activity. This may be due to the PAH destruction or enhanced VSG continuum by the central AGN.

\section{ACKNOWLEDGEMENTS}

We thank Jia-sheng Huang, Zhong Wang, David Sanders, and Yan-Chun Liang for helpful discussions. Also many thanks to anonymous referees. H.W. is grateful to the Institute for Astronomy, University of Hawaii for hospitality. This work was supported by the National Science Foundation of China under grants 10273012, 10333060, and 10473013,

and a CAS grant KJCX3-SYW-N2. It is, in part, based on observations made with the Spitzer Space Telescope, which is operated by Jet Propulsion Laboratory of the California Institute of Technology under NASA Contract 1407.

Funding for the SDSS and SDSS-II has been provided by the Alfred P. Sloan Foundation, the Participating Institutions, the National Science Foundation, the U.S. Department of Energy, the National Aeronautics and Space Administration, the Japanese Monbukagakusho, the Max Planck Society, and the Higher Education Funding Council for England. The SDSS Web Site is http://www.sdss.org/,

The SDSS is managed by the Astrophysical Research Consortium for the Participating Institutions. The Participating Institutions are the American Museum of Natural History, Astrophysical Institute Potsdam, University of Basel, University of Cambridge, Case Western Reserve University, University of Chicago, Drexel University, Fermilab, the Institute for Advanced Study, the Japan Participation Group, Johns Hopkins University, the Joint Institute for Nuclear Astrophysics, the Kavli Institute for Particle Astrophysics and Cosmology, the 
Korean Scientist Group, the Chinese Academy of Sciences (LAMOST), Los Alamos National Laboratory, the Max-Planck-Institute for Astronomy (MPIA), the Max-Planck-Institute for Astrophysics (MPA), New Mexico State University, Ohio State University, University of Pittsburgh, University of Portsmouth, Princeton University, the United States Naval Observatory, and the University of Washington.

\section{REFERENCES}

Adelman-McCarthy, J. K., et al. 2006, ApJS, 162, 38

Aitken, D. K., \& Roche, P. F. 1985, MNRAS, 213, 777

Alonso-Herrero, A., Rieke, G. H., Rieke, M. J., Colina, L., Pérez-González, P. G., \& Ryder, S. D. 2006, ApJ, 650, 835

Armus, L., et al. 2007, ApJ, 656, 148

Bertin, E., \& Arnouts, S. 1996, A\&AS, 117, 393

Bohlin, R. C., Savage, B. D., \& Drake, J. F. 1978, ApJ, 224, 132

Boselli, A., Lequeux, J., \& Gavazzi, G. 2004, A\&A, 428, 409

Bruzual, G., \& Charlot, S. 2003, MNRAS, 344, 1000

Calzetti, D., et al. 2005, ApJ, 633, 871

Calzetti, D. 2001, PASP, 113, 1449

Cao, C., \& Wu, H. 2007, AJ, 133, 1710

Contursi, A., et al. 2000, A\&A, 362, 310

Cutri, R. M., et al. 2003, The IRSA 2MASS All-Sky Point Source Catalog, NASA/IPAC

Dale, D. A., et al. 2006, ApJ, 646, 161

Draine, B. T., \& Li, A. 2007, ApJ, 657, 810

Draine, B. T., et al. 2007, ArXiv Astrophysics e-prints, arXiv:astro-ph/0703213

Draine, B. T. 2003, ARA\&A, 41, 241

Dwek, E. 1998, ApJ, 501, 643 
Elbaz, D., Cesarsky, C. J., Chanial, P., Aussel, H., Franceschini, A., Fadda, D., \& Chary, R. R. 2002, A\&A, 384, 848

Engelbracht, C. W., Gordon, K. D., Rieke, G. H., Werner, M. W., Dale, D. A., \& Latter, W. B. 2005, ApJ, 628, L29

Fazio, G. G. et al., 2004, ApJS, 154, 10

Förster Schreiber, N. M., Roussel, H., Sauvage, M., \& Charmandaris, V. 2004, A\&A, 419, 501

Galliano, F., Madden, S. C., Jones, A. P., Wilson, C. D., \& Bernard, J.-P. 2005, A\&A, 434, 867

Galliano, F. 2006, ArXiv Astrophysics e-prints, arXiv:astro-ph/0610852

Genzel, R., \& Cesarsky, C. J. 2000, ARA\&A, 38, 761

Hancock, M., Smith, B. J., Struck, C., Giroux, M. L., Appleton, P. N., Charmandaris, V., \& Reach, W. T. 2007, AJ, 133, 676

Hogg, D. W., Tremonti, C. A., Blanton, M. R., Finkbeiner, D. P., Padmanabhan, N., Quintero, A. D., Schlegel, D. J., \& Wherry, N. 2005, ApJ, 624, 162

Houck, J. R., et al. 2004a, ApJS, 154, 18

Houck, J. R., et al. 2004b, ApJS, 154, 211

Huang, J.-S., et al. 2004, ApJS, 154, 44

Kauffmann, G., et al. 2003, MNRAS, 346, 1055

Kennicutt, R. C., Jr., et al. 2003, PASP, 115, 928

Kewley, L. J., Dopita, M. A., Sutherland, R. S., Heisler, C. A., \& Trevena, J. 2001, ApJ, 556,121

Kewley, L. J., Groves, B., Kauffmann, G., \& Heckman, T. 2006, MNRAS, 372, 961

Lacy, M., et al. 2005, ApJS, 161, 41

Laurent, O., Mirabel, I. F., Charmandaris, V., Gallais, P., Madden, S. C., Sauvage, M., Vigroux, L., \& Cesarsky, C. 2000, A\&A, 359, 887

Lonsdale, C. J., et al. 2003, PASP, 115, 897 
Madden, S. C. 2000, New Astronomy Review, 44, 249

Madden, S. C., Galliano, F., Jones, A. P., \& Sauvage, M. 2006, A\&A, 446, 877

Oke, J. B., \& Gunn, J. E. 1983, ApJ, 266, 713

Pagel, B. E. J., \& Edmunds, M. G. 1981, ARA\&A, 19, 77

Rieke, G. H., et al. 2004, ApJS, 154, 25

Roche, P. F., Aitken, D. K., Smith, C. H., \& Ward, M. J. 1991, MNRAS, 248, 606

Roche, P. F., \& Aitken, D. K. 1985, MNRAS, 213, 789

Rosenberg, J. L., Ashby, M. L. N., Salzer, J. J., \& Huang, J.-S. 2006, ApJ, 636, 742

Roussel, H., Sauvage, M., Vigroux, L., \& Bosma, A. 2001, A\&A, 372, 427

Siebenmorgen, R., Krügel, E., \& Spoon, H. W. W. 2004, A\&A, 414, 123

Smith, B. J., Struck, C., Hancock, M., Appleton, P. N., Charmandaris, V., \& Reach, W. T. 2007a, AJ, 133, 791

Smith, J. D. T., et al. 2007b, ApJ, 656, 756

Smith, J. A., et al. 2002, AJ, 123, 2121

Stoughton, C., et al. 2002, AJ, 123, 485

Sturm, E., Lutz, D., Verma, A., Netzer, H., Sternberg, A., Moorwood, A. F. M., Oliva, E., \& Genzel, R. 2002, A\&A, 393, 821

Surace J. A., et al., 2005, The SWIRE Data Release 2: Image Atlases and Source Catalogs for ELAIS-N1, ELAIS-N2, XMM-LSS, and the Lockman Hole

Thornley, M. D., Schreiber, N. M. F., Lutz, D., Genzel, R., Spoon, H. W. W., Kunze, D., \& Sternberg, A. 2000, ApJ, 539, 641

Thuan, T. X., Sauvage, M., \& Madden, S. 1999, ApJ, 516, 783

Tremonti, C. A., et al. 2004, ApJ, 613, 898

Veilleux, S., \& Osterbrock, D. E. 1987, ApJS, 63, 295

Verma, A., Charmandaris, V., Klaas, U., Lutz, D., \& Haas, M. 2005, Space Science Reviews, 119,355 
Vogler, A., Madden, S. C., Beck, R., Lundgren, A. A., Sauvage, M., Vigroux, L., \& Ehle, M. 2005, A\&A, 441, 491

Weedman, D. W., et al. 2005, ApJ, 633, 706

Wen, X. Q., Wu, H., Cao, C., Xia, X. Y., 2007, ChJAA, 7, 187

Werner, M. W. et al., 2004, ApJS, 154, 1

Wu, H., Zou, Z. L., Xia, X. Y., \& Deng, Z. G. 1998, A\&AS, 132, 181

Wu, H., Cao, C., Hao, C.-N., Liu, F.-S., Wang, J.-L., Xia, X.-Y., Deng, Z.-G., \& Young, C. K.-S. 2005, ApJ, 632, L79

Wu, Y., Charmandaris, V., Hao, L., Brandl, B. R., Bernard-Salas, J., Spoon, H. W. W., \& Houck, J. R. 2006, ApJ, 639, 157 


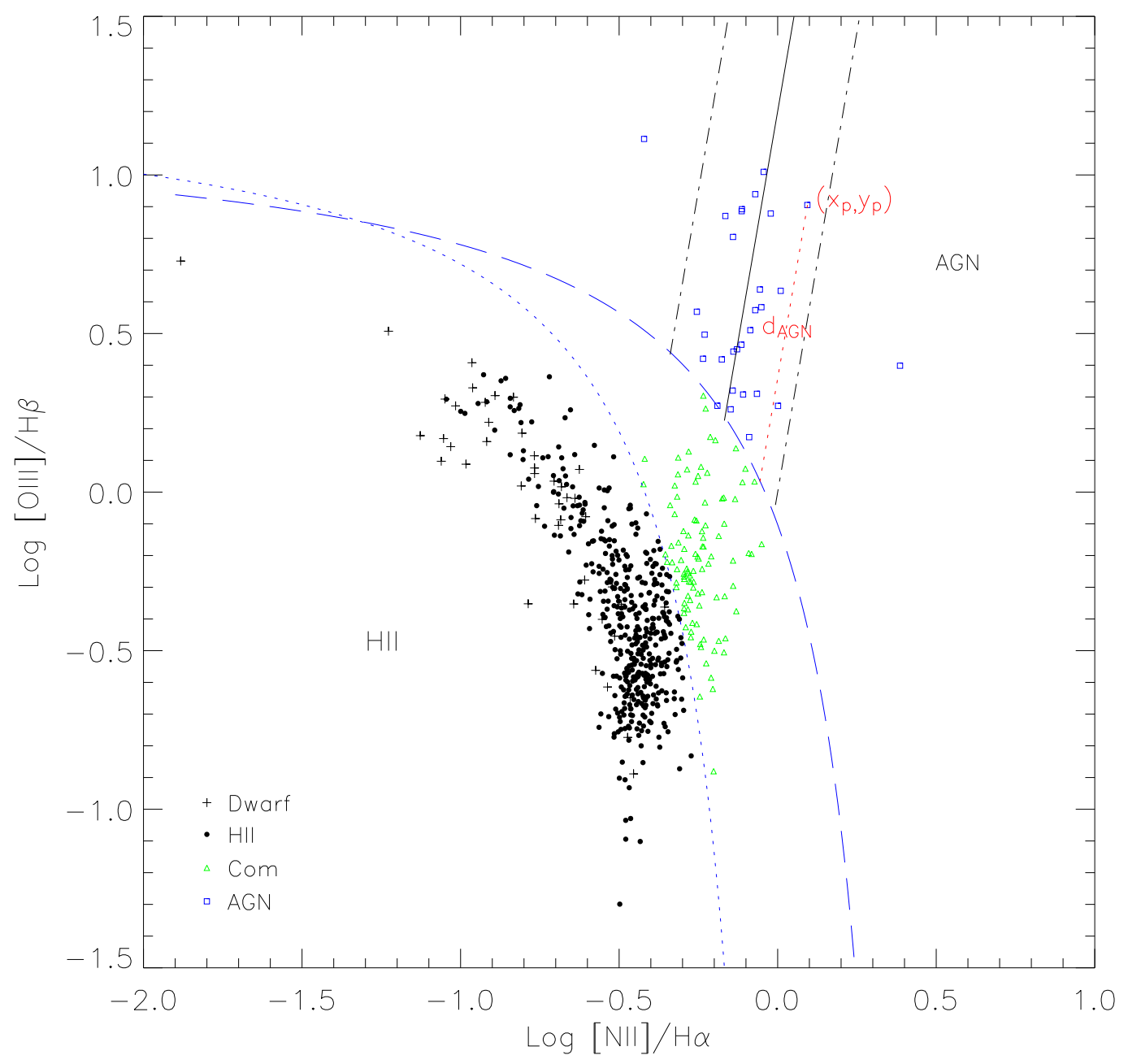

Fig. 1. - The line-diagnostic diagram $[\mathrm{OIII}] / \mathrm{H} \beta$ versus $[\mathrm{NII}] / \mathrm{H} \alpha$. The dotted curve is the parametrized curve by Kauffmann et al. (2003) and the dashed curve is the theoretical boundary for starburst (Kewley et al. 2001). Under the dotted curve, the HII galaxies are labelled as solid circles and dwarf galaxies as pluses. The composites are between the dotted and dashed curves and are labelled as open triangles. The AGNs are above the dashed curve and are labelled as open boxes. To characterize AGN activity, we define the AGN distance $d_{A G N}$ as the distance from these boxes to the dashed curve along the best-fit solid line. 


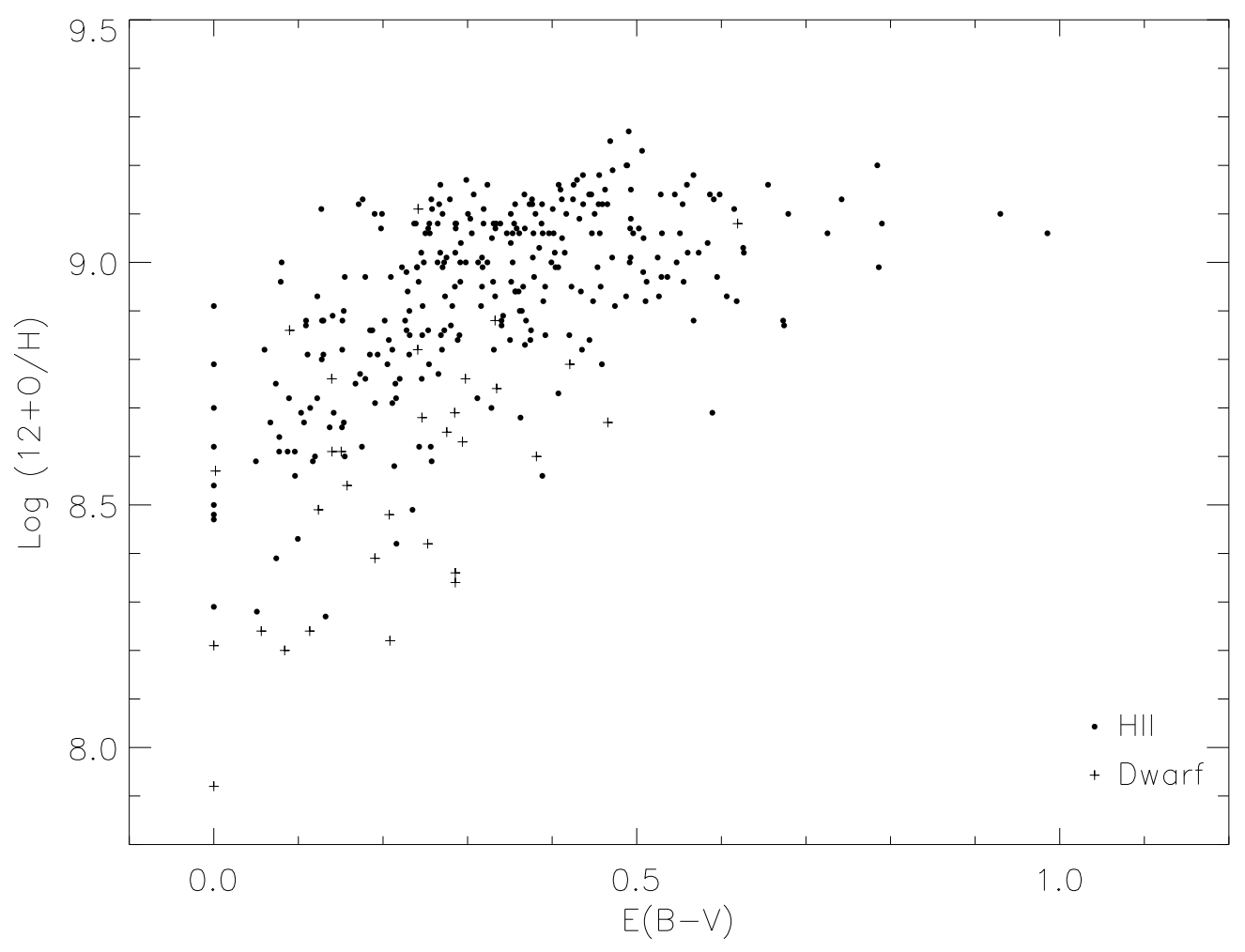

Fig. 2.- The metallicities of HII galaxies as a function of their internal reddening. The plus symbols are dwarf galaxies. 


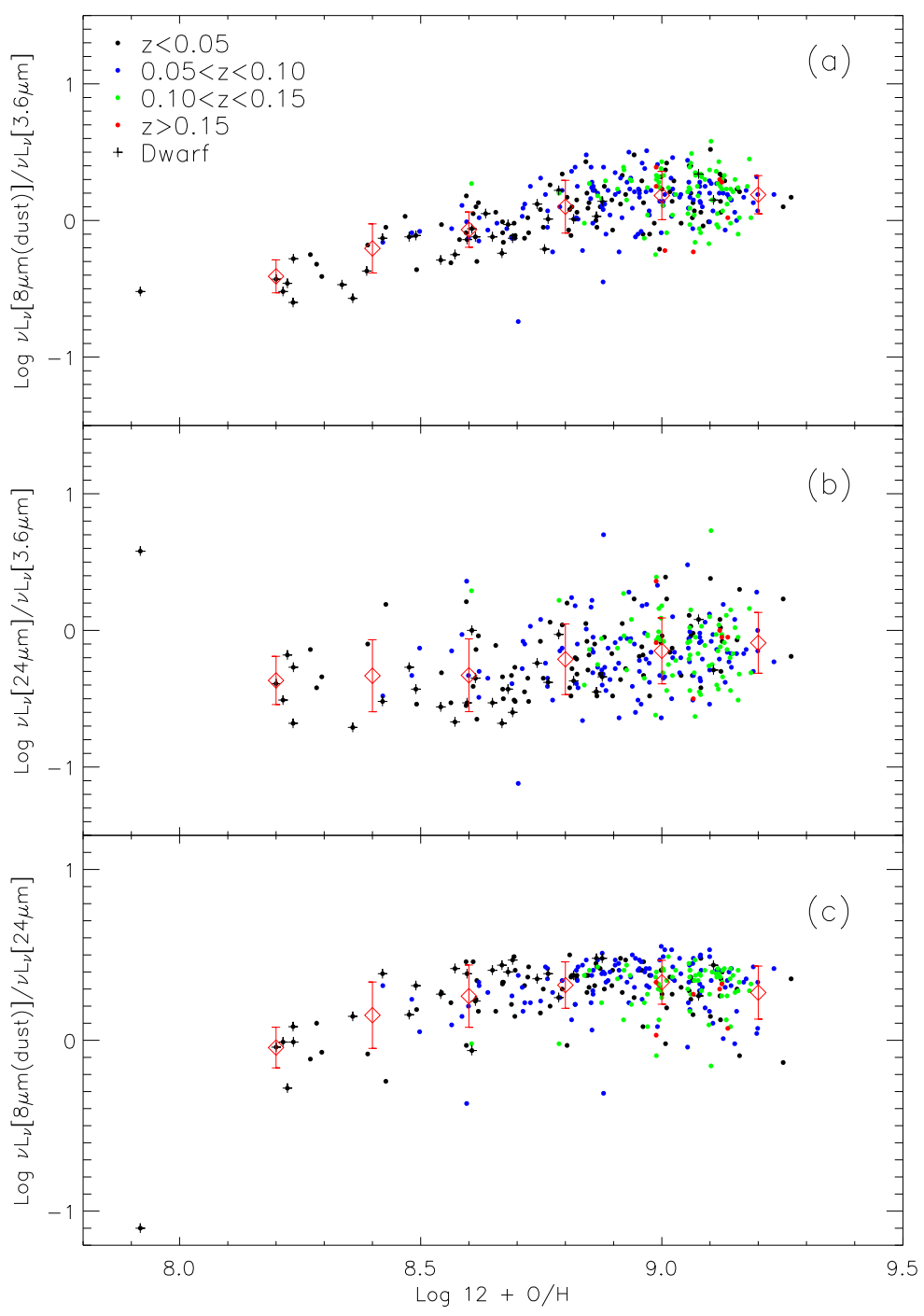

Fig. 3.- The MIR ratios (a) $\nu L_{\nu}[8 \mu m(d u s t)] / \nu L_{\nu}[3.6 \mu m]$, ] (b) $\nu L_{\nu}[24 \mu m] / \nu L_{\nu}[3.6 \mu m]$, and (c) $\nu L_{\nu}[8 \mu m(d u s t)] / \nu L_{\nu}[24 \mu m]$ versus metallicity. The plus symbols represent the dwarf galaxies and the solid circles are normal galaxies. The diamonds and bars give the mean values and 1- $\sigma$ standard deviations with a metallicity bin of 0.2 . Different colors show galaxies in different redshift bins. 


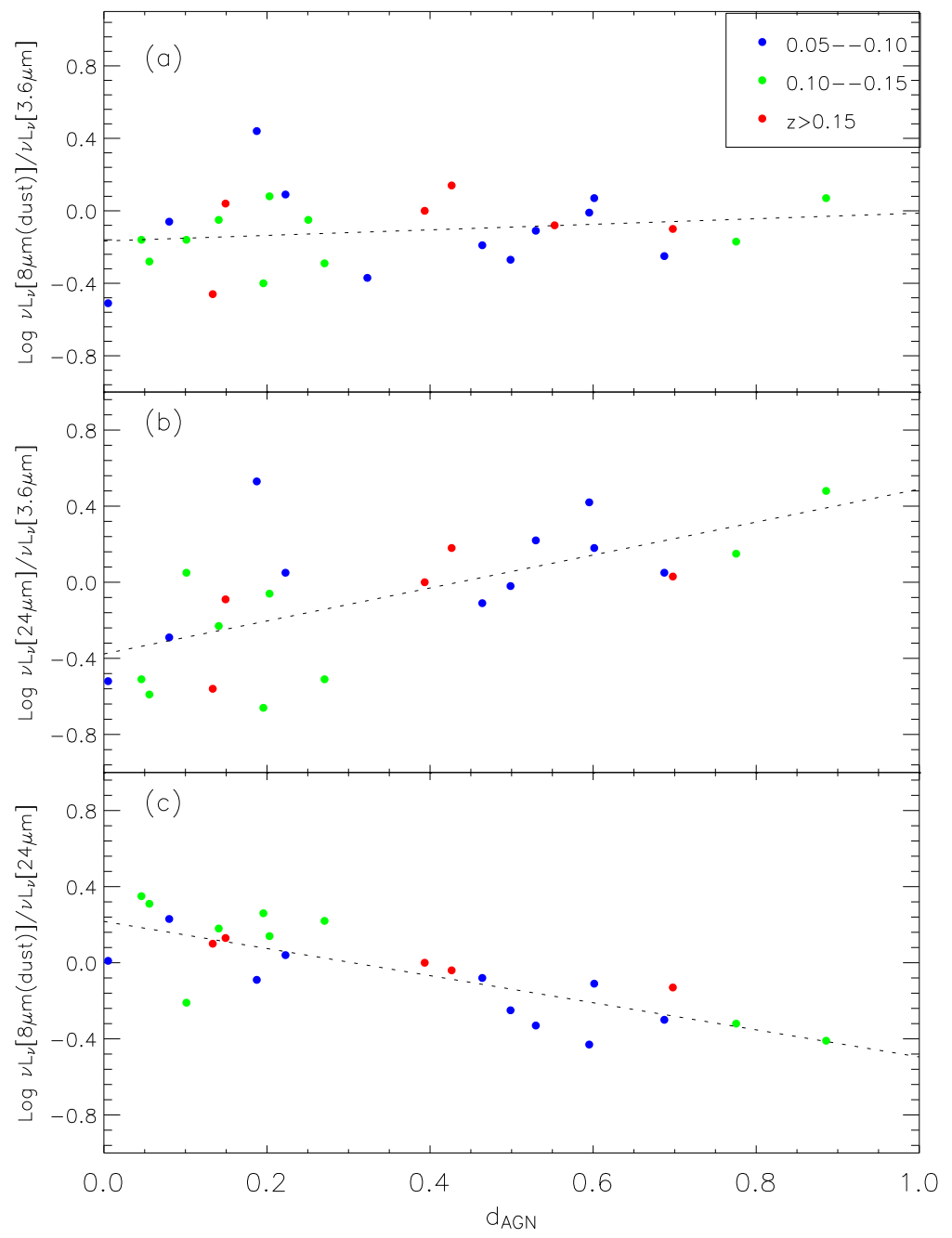

Fig. 4. - The MIR ratios (a) $\nu L_{\nu}\left[8 \mu m(\right.$ dust) $] / \nu L_{\nu}[3.6 \mu m]$, (b) $\nu L_{\nu}[24 \mu m] / \nu L_{\nu}[3.6 \mu m]$, and (c) $\nu L_{\nu}[8 \mu m(d u s t)] / \nu L_{\nu}[24 \mu m]$ versus the distance $d_{A G N}$. Different colors represent AGNs in different redshift bins. The dotted lines represent the best linear fitting. 


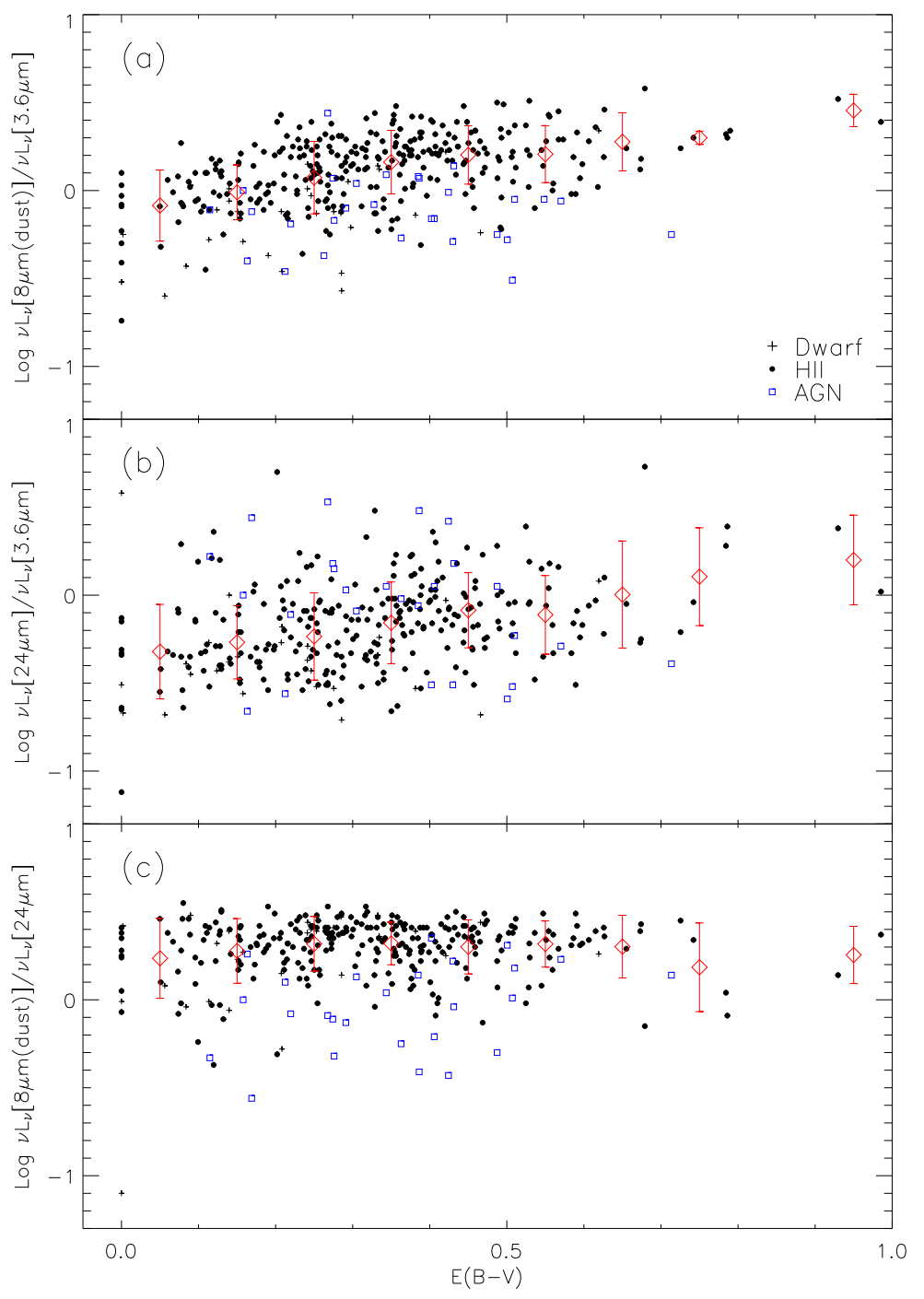

Fig. 5. - The MIR ratios (a) $\nu L_{\nu}\left[8 \mu m(\right.$ dust) $] / \nu L_{\nu}[3.6 \mu m]$, (b) $\nu L_{\nu}[24 \mu m] / \nu L_{\nu}[3.6 \mu m]$, and (c) $\nu L_{\nu}[8 \mu m(d u s t)] / \nu L_{\nu}[24 \mu m]$ as functions of reddening. The symbols are the same as in Figure 1. The diamonds and bars give the mean values and 1- $\sigma$ standard deviations of HII galaxies with an $\mathrm{E}(\mathrm{B}-\mathrm{V})$ bin of 0.1 . 


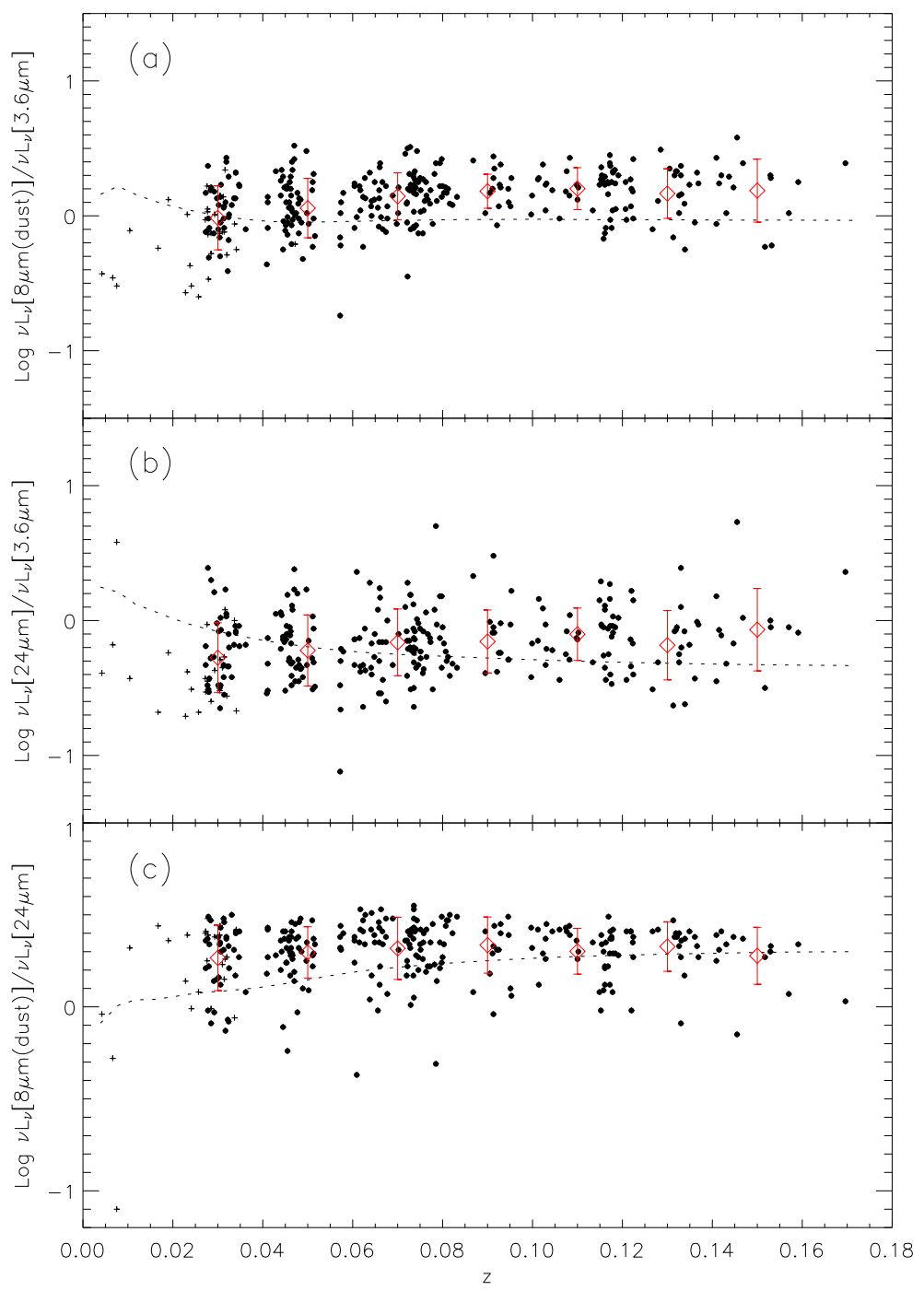

Fig. 6. - The MIR ratios (a) $\nu L_{\nu}\left[8 \mu m(\right.$ dust) $] / \nu L_{\nu}[3.6 \mu m]$, (b) $\nu L_{\nu}[24 \mu m] / \nu L_{\nu}[3.6 \mu m]$, and (c) $\nu L_{\nu}[8 \mu m(d u s t)] / \nu L_{\nu}[24 \mu m]$ as functions of redshift. The diamonds and bars are the mean values and 1- $\sigma$ standard deviations with a redshift bin of 0.02 . The dotted curves show the variation of the MIR ratios of NGC 3351 with redshift (aperture). Dward galaxies are labelled as plus symbols. 


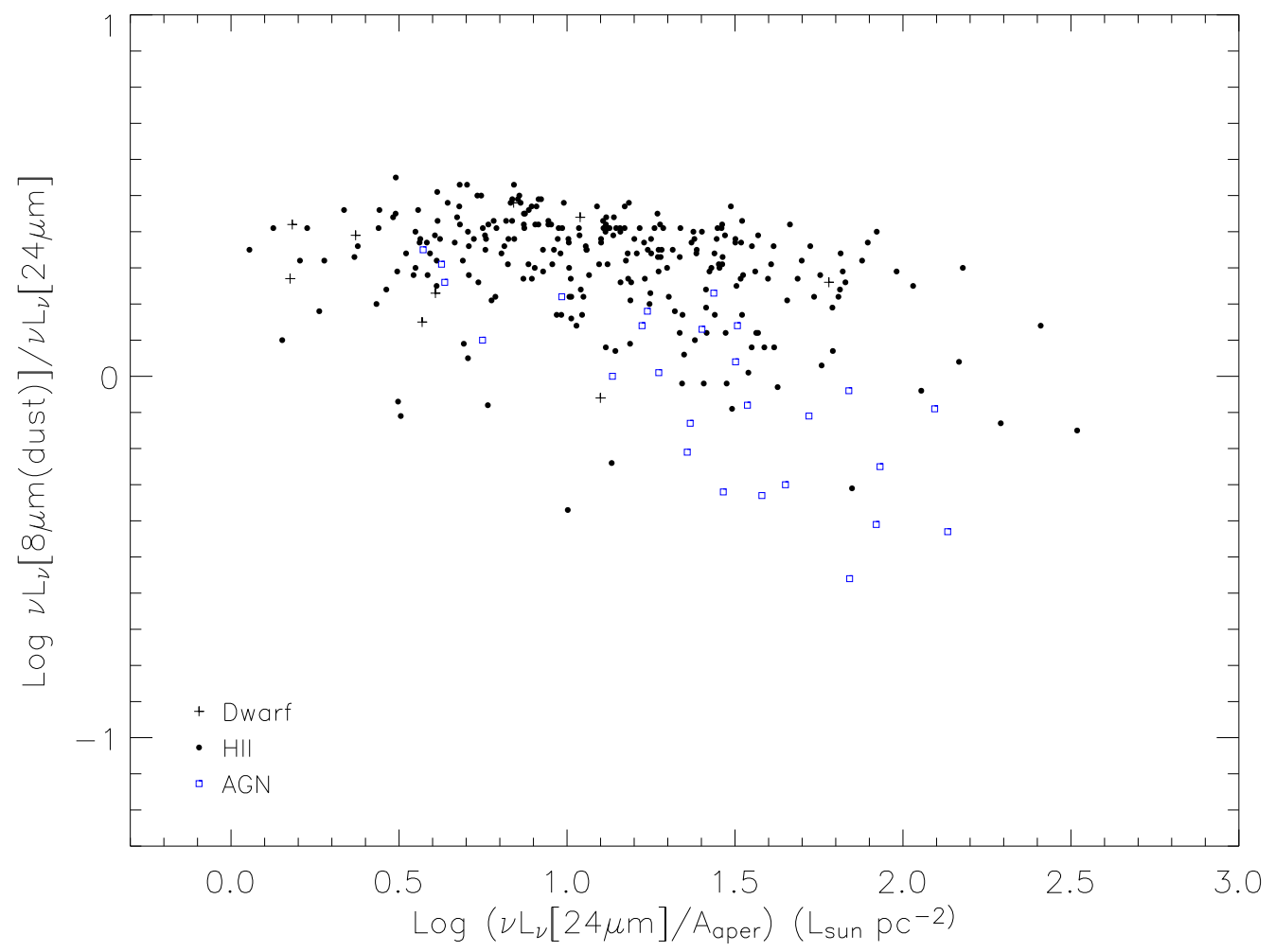

Fig. 7.- The MIR ratio $\nu L \nu[8 \mu m(d u s t)] / \nu L \nu[24 \mu m]$ as a function of the $24 \mu \mathrm{m}$ intensity. The $24 \mu \mathrm{m}$ intensity is defined as the $24 \mu \mathrm{m}$ luminosity in unit physical area and is obtained from the ratio of the $24 \mu \mathrm{m}$ luminosity to the physical area of aperture 6 " in each galaxy. The symbols are the same as in Figure 1. 


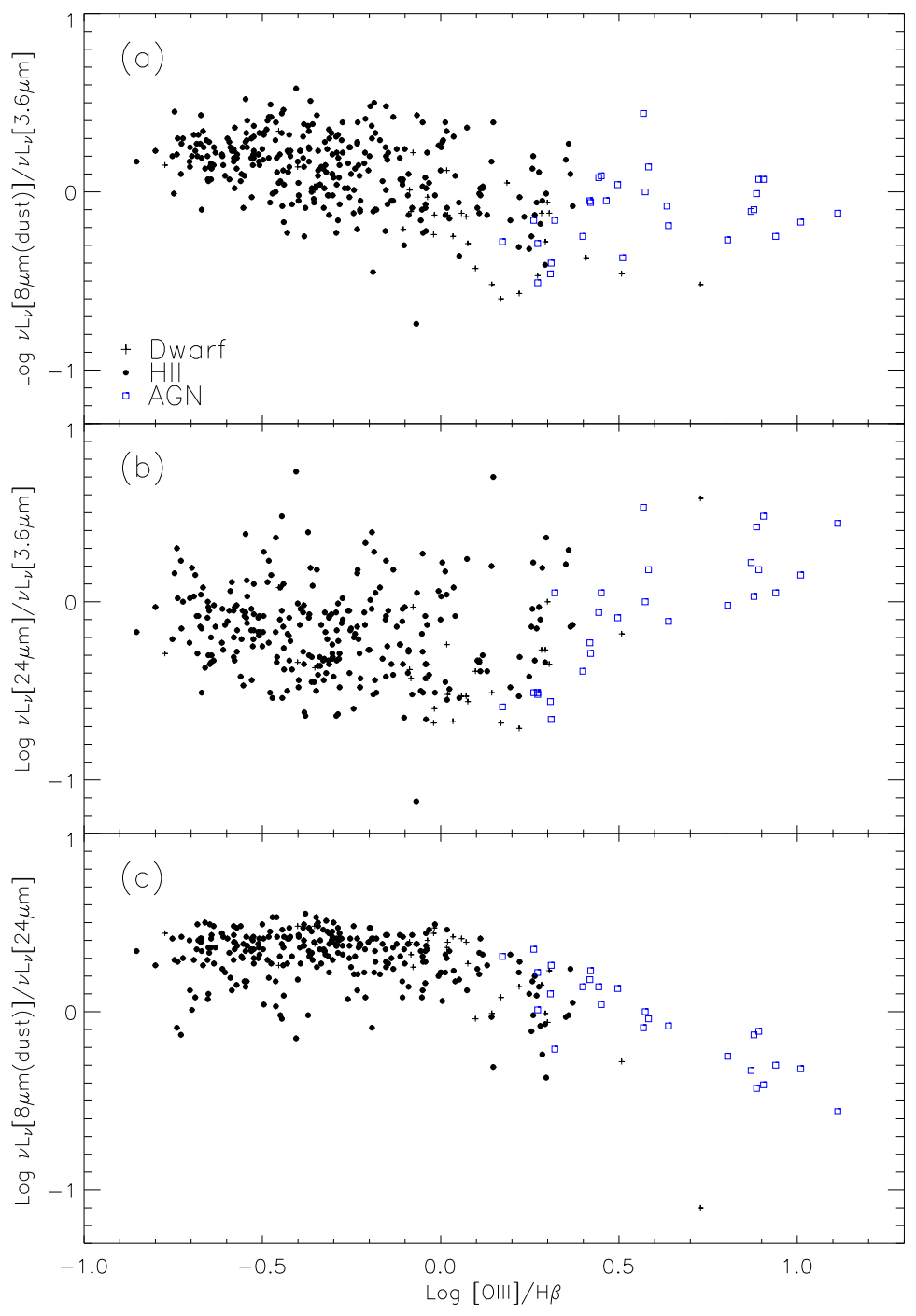

Fig. 8. - The MIR ratios (a) $\nu L_{\nu}\left[8 \mu m(\right.$ dust) $] / \nu L_{\nu}[3.6 \mu m]$, (b) $\nu L_{\nu}[24 \mu m] / \nu L_{\nu}[3.6 \mu m]$, and (c) $\nu L_{\nu}[8 \mu m(d u s t)] / \nu L_{\nu}[24 \mu m]$ as functions of the emission line ratio $\log ([\mathrm{OIII}] / \mathrm{H} \beta)$. The symbols are the same as in Figure 1 . 